\title{
Shape Analysis of 3D Head Scan Data for U.S. Respirator Users
}

\author{
Ziqing Zhuang, ${ }^{1}$ Dennis E. Slice, ${ }^{2}$ Stacey Benson, ${ }^{3}$ Stephanie Lynch, ${ }^{1}$ and Dennis J. Viscusi ${ }^{1}$ \\ ${ }^{1}$ National Personal Protective Technology Laboratory, National Institute for Occupational Safety and Health, \\ Pittsburgh, PA 15236, USA \\ ${ }^{2}$ Department of Scientific Computing, Florida State University, Dirac Science Library, Tallahassee, FL 32306-4120, USA \\ ${ }^{3}$ EG\&G Technical Services Inc., Pittsburgh, PA 15236, USA
}

Correspondence should be addressed to Ziqing Zhuang, zaz3@cdc.gov

Received 25 November 2009; Accepted 29 January 2010

Academic Editor: Yingzi Du

Copyright ( 2010 Ziqing Zhuang et al. This is an open access article distributed under the Creative Commons Attribution License, which permits unrestricted use, distribution, and reproduction in any medium, provided the original work is properly cited.

In 2003, the National Institute for Occupational Safety and Health (NIOSH) conducted a head-and-face anthropometric survey of diverse, civilian respirator users. Of the 3,997 subjects measured using traditional anthropometric techniques, surface scans and 26 three-dimensional (3D) landmark locations were collected for 947 subjects. The objective of this study was to report the size and shape variation of the survey participants using the 3D data. Generalized Procrustes Analysis (GPA) was conducted to standardize configurations of landmarks associated with individuals into a common coordinate system. The superimposed coordinates for each individual were used as commensurate variables that describe individual shape and were analyzed using Principal Component Analysis (PCA) to identify population variation. The first four principal components (PC) account for $49 \%$ of the total sample variation. The first PC indicates that overall size is an important component of facial variability. The second PC accounts for long and narrow or short and wide faces. Longer narrow orbits versus shorter wider orbits can be described by PC3, and PC4 represents variation in the degree of ortho/prognathism. Geometric Morphometrics provides a detailed and interpretable assessment of morphological variation that may be useful in assessing respirators and devising new test and certification standards.

\section{Introduction}

Millions of workers across the United States depend on respirators for personal protection everyday. Respirators have to fit to provide adequate protection to these workers. Assessing respirator fit has for many years been based on fit test panels from Air Force data from the 1970s [1, 2]. Given an array of respirator styles and sizes, it is important to determine their fit and efficacy with respect to their intended user population and to quantify those facial features relevant to fit. It is largely recognized that data based on a population of young, healthy military personnel from over 30 years ago are not likely to be representative of the diversity of the contemporary workforce that fit test panels should target [3]. To address this deficiency, the National Institute for Occupational Safety and Health (NIOSH) conducted a facial morphological survey of contemporary workers that require the use of a respirator in the course of their work $[4,5]$.

Besides being based on a group not likely to be completely representative of the contemporary respirator-user population, previous studies focused on the association between linear facial dimensions in the development of test panels to capture facial variation. In the field of anthropometrics, from which the facial measurements were borrowed, there has been considerable recent innovation in the quantification and statistical analysis of shapes based on the study of the Cartesian coordinates of the landmarks that usually serve as the basis for traditional measurement definitions $[6,7]$. These new methods, collectively referred to as Geometric Morphometrics (GMs), have proven more powerful and efficient than traditional approaches in many cases, and it is worthwhile to determine the extent to which they can advance the goal of respirator fit assessment. Such studies, in turn, could feed back into respirator design to achieve more efficient and comfortable product style and sizing. In anticipation of this, the NIOSH study included the collection of both facial surface scans and three-dimensional landmark locations for a large subset $(\sim 25 \%)$ of their surveyed individuals [4].

The dependence of respirator-fit assessment standards on a base population morphologically distinct from the target 
population and the reliance of the development of those standards on a limited and somewhat arbitrary suite of traditional; (curvi-) linear anthropometric measurements were some of the problems identified by an independent review committee that examined the current state of respirator-fit assessment [8]. It was the purpose of this study to address some of these concerns by further investigating the nature of facial shape variation in the latest data assembled using GM techniques.

\section{Materials and Methods}

2.1. Data. Data for this study were obtained from the NIOSH National Personal Protective Technology Laboratory (NPPTL) facial anthropometric survey [4]. The main body of data consisted of 947 data files in the format of a Unixbased, 3D package called INTEGRATE [9]. Each file contained three-dimensional coordinate locations of anatomical landmarks (Figure 1) for one individual. In addition, demographic information including sex, age group, racial group, and traditional anthropometric measures were collected. All data were visually inspected using morphometrics software to identify mislabeled or obviously erroneous coordinate values. These were marked as missing data.

The proper handling of missing data is a complicated endeavor [10]. One possible course of action would be to eliminate all individuals with any missing landmarks. That would call for the removal of over $25 \%$ of the data set, which seems extreme. Several other cut points would be defensible, for example, removing individuals with more than 3 missing landmarks, 5, and so forth. It was decided, instead, to retain all 947 individuals. Most individuals (72\%) had no missing landmark coordinates, and less than $1 \%$ had six or more missing landmarks out of the twenty-eight with missing data. If the occurrence of missing data is not random with respect to the morphology of the individuals, then removing individuals will reduce the variability that this study is seeking to quantify. Missing data were estimated by simply substituting mean coordinate values.

2.2. Generalized Procrustes Analysis. Landmark coordinates are not directly comparable as quantitative measures of shape because they are (usually) recorded with respect to an arbitrary set of orthogonal reference axes. In its simplest case, irrelevant variation is introduced into the coordinate values by the position and orientation of the specimen relative to the digitizing apparatus or scanning device. In addition, many standard morphometric analyses, using both traditional measurements and landmark coordinates, seek to sequester size variation, which often tends to dominate sample variability, into a separate variable. To address these problems and issues, geometric morphometric methods include a data processing step that standardizes configurations of landmarks associated with individuals into a common coordinate system and, further, usually standardizes these configurations to a common size. The scale factor used in the latter standardization can be saved as a size measure for further investigations of the relationship between shape and size in the sample.

The way the required standardization is usually done is through Generalized Procrustes Analysis (GPA) $[6,11,12]$. In GPA, landmark configurations are mean-centered so that their average coordinate location for all landmarks is the origin. They are then scaled so that the square root of the sum of squared distances of each landmark in a configuration to their joint average location (the origin after mean-centering) is 1.0. This measure is called centroid size and has the desirable property that it is the only size measure that is independent of shape variation in the presence of small, isometric random variation in landmark location around a mean configuration [13]. Next, an arbitrary configuration of landmarks from the mean-centered and size-standardized data set (usually the first specimen) is used as a reference configuration. All specimens in the data are rotated so that the sum of squared distances between individual configuration landmarks and corresponding landmarks on the reference is minimized. Once so rotated, a mean configuration is estimated as the arithmetic averages of landmark coordinates in the superimposed data set. The average configuration is then scaled to unit centroid size and the sample refit to the new estimated mean. This process is guaranteed to monotonically converge on a mean estimate for the sample [11] and is not substantively affected by the initial choice of reference. After little or no change is seen due to the rotation and mean estimation steps, the process is deemed complete and the superimposed coordinates for each individual can be used as commensurate variables that describe individual shape and can be subjected to multivariate analyses, such as principal components analysis used here.

This approach, in its standard form, is not the best for the purposes of this study directed at assessing variability that influences the fit and function of respirators. Here, size variation is not less important to the ultimate goal than shape variation, and even sequestering it in a separate variable for joint or separate analysis is, at least initially, irrelevant. For this reason, scale was restored to the results of a standard GPA by multiplying the resulting shape variables by the inverse of the scale factor applied to them in the course of the superimposition of individual configurations onto the grand mean. These are the "form" (shape + size) data used in subsequent statistical analyses of population variation.

2.3. Population Variation. Population variation for the data set, after GPA, was analyzed by principal components analysis (PCA) to identify patterns of covariation in the data. Major directions of variation were compared and visualized using GM methods and software.

2.4. Software. The above analyses were carried out using a combination of standard statistical software, existing morphometrics software, and new routines developed specifically for analyzing the data used in the study. All standard statistical analyses, such as PCA, were carried out in the open source $\mathrm{R}$ package [14]. The matrix capabilities of $\mathrm{R}$ were also used for some custom data manipulation and testing. 

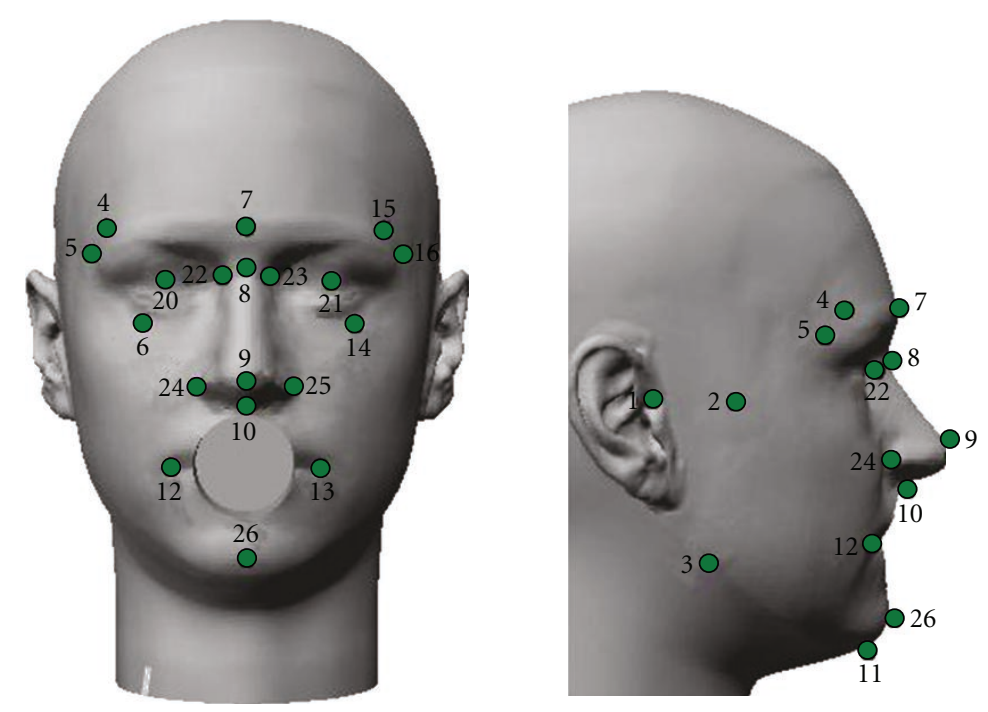

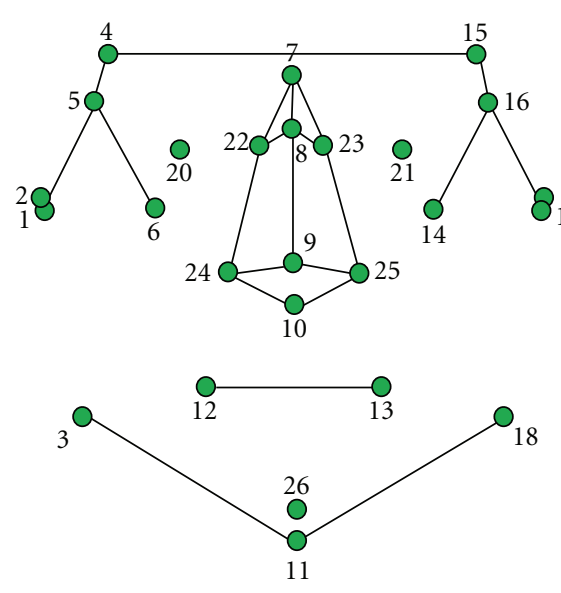

(a)
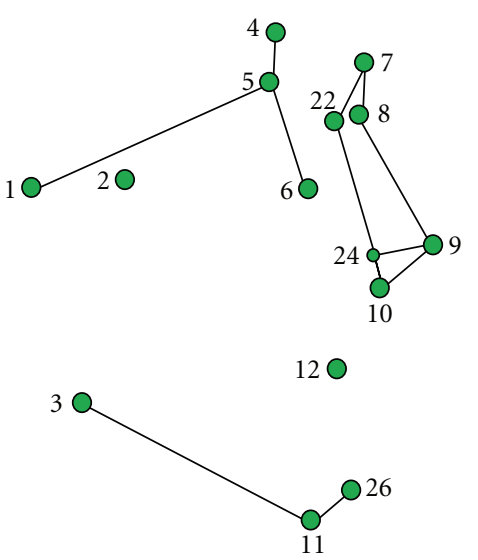

(b)

Figure 1: Location and identification of the 26 landmarks used for the PCA: Tragion $(1,19)$, Zygion $(2,17)$, gonion $(3,18)$, Frontotemporale $(4,15)$, Zygofrontale (5, 16), Infraorbitale (6, 14), Glabella (7), Sellion (8), Pronasale (9), Subnasale (10), Menton (11), Chelion (12, 13), Pupil (20, 21), Nasal root point (22, 23), Alare (24, 25), and Chin (26).

Where possible, new Java-based, cross-platform programs ( $\mathrm{m}_{-}$vis and the new of Morpheus et al.) currently under development by one of the authors (Slice) were used for visualization and data manipulation and analysis. A number of new routines were added to these programs to facilitate the current study. When morphometric-specific visualization or analytical routines were not available in the most recent versions of this software, an older Microsoft Windows version of Morpheus et al., written in C++, was used [15].

\section{Results}

Figure 2 shows the data set extracted from the GPA with size restoration as described above. Each cluster of symbols represents the scatter of individual landmark locations for the 947 individuals in this data set. The coordinates of the 26 landmarks per individual represented in Figure 2 are a slightly redundant set of 78 ( 26 points $\times 3$ coordinates per point) form variables that characterize the size and shape of individual faces within a coordinate system common to all.

Principal component analysis of the 947 superimposed configurations in the space of the 78 form variables showed a substantial proportion of the total sample variability in the first four PCs $(26 \%, 10 \%, 8 \%$, and 5\%, resp.). The variance on PCs beyond the third (all 5\% or less of the total) trail off gradually suggesting no strong patterns of intercorrelation amongst the variables. Nonetheless, the first two PCs together only represent $36 \%$ of total sample variability and the first four only $49 \%$. In fact, it requires the first 27 PCs as a group to account for $90 \%$ of total sample variation. This suggests that the bivariate approach used in constructing fit panels may be ignoring a substantial and important aspect of total sample variability.

The Eigenvectors for each PC are used to multiply the superimposed coordinates to obtain the scores for each PC. 
TABle 1: Eigenvectors from Principle Component Analysis.

\begin{tabular}{|c|c|c|c|c|c|}
\hline Face Dimensions & & PC1 & PC2 & PC3 & PC4 \\
\hline \multirow{3}{*}{ Right Tragion } & $x$ & -0.092402 & -0.09838 & -0.082324 & 0.124948 \\
\hline & $y$ & 0.055546 & -0.03336 & -0.000476 & -0.046974 \\
\hline & $z$ & -0.226846 & -0.015639 & -0.081106 & 0.103059 \\
\hline \multirow{3}{*}{ Right Bizigomatic } & $x$ & -0.105446 & -0.037155 & -0.250892 & 0.06538 \\
\hline & $y$ & 0.058617 & -0.021507 & -0.008662 & 0.046299 \\
\hline & $z$ & -0.264815 & -0.218134 & 0.230527 & 0.146395 \\
\hline \multirow{3}{*}{ Right Bigonion } & $x$ & -0.175016 & -0.170624 & -0.077249 & -0.010609 \\
\hline & $y$ & -0.153711 & 0.283158 & 0.025585 & 0.251424 \\
\hline & $z$ & -0.236316 & -0.052436 & -0.174264 & -0.223689 \\
\hline \multirow{3}{*}{ Right Frontotemporale } & $x$ & -0.087244 & -0.058213 & -0.171941 & 0.181386 \\
\hline & $y$ & 0.185991 & -0.083649 & -0.231942 & 0.088256 \\
\hline & $z$ & -0.065722 & 0.041468 & -0.118217 & -0.008149 \\
\hline \multirow{3}{*}{ Right Zygofrontale } & $x$ & -0.124784 & -0.061425 & -0.131633 & 0.128663 \\
\hline & $y$ & 0.099636 & -0.079601 & -0.122976 & 0.12982 \\
\hline & $z$ & -0.049474 & 0.035962 & -0.217425 & -0.005845 \\
\hline \multirow{3}{*}{ Right Infraorbitale } & $x$ & -0.155163 & -0.153839 & 0.1021 & -0.008096 \\
\hline & $y$ & 0.002082 & -0.081024 & 0.071887 & -0.030466 \\
\hline & $z$ & -0.084867 & -0.135606 & 0.177772 & 0.031694 \\
\hline \multirow{3}{*}{ Glabella } & $x$ & -0.031366 & -0.009799 & 0.004402 & 0.088434 \\
\hline & $y$ & 0.07538 & -0.246936 & 0.075979 & 0.015563 \\
\hline & $z$ & 0.065039 & 0.032831 & -0.023948 & -0.165925 \\
\hline \multirow{3}{*}{ Sellion } & $x$ & -0.024137 & 0.000893 & -0.000995 & 0.071671 \\
\hline & $y$ & 0.054995 & -0.202543 & 0.014098 & -0.056409 \\
\hline & $z$ & 0.046018 & 0.007447 & -0.028295 & -0.150108 \\
\hline \multirow{3}{*}{ Pronasale } & $x$ & -0.048801 & 0.006432 & 0.009248 & 0.016389 \\
\hline & $y$ & -0.021646 & 0.023393 & 0.066119 & 0.015546 \\
\hline & $z$ & 0.098337 & -0.077382 & -0.050498 & -0.070001 \\
\hline \multirow{3}{*}{ Subnasale } & $x$ & -0.041782 & 0.003084 & 0.003778 & -0.032842 \\
\hline & $y$ & -0.03442 & 0.021448 & 0.078113 & -0.003448 \\
\hline & $z$ & 0.090048 & -0.049884 & -0.013582 & 0.052307 \\
\hline \multirow{3}{*}{ Menton } & $x$ & -0.026572 & -0.075211 & 0.009377 & -0.04029 \\
\hline & $y$ & -0.239456 & 0.282161 & 0.064054 & -0.137788 \\
\hline & $z$ & 0.072425 & 0.131381 & -0.022598 & 0.095107 \\
\hline \multirow{3}{*}{ Right Chelion } & $x$ & -0.104235 & -0.011205 & -0.065256 & -0.122249 \\
\hline & $y$ & -0.122439 & 0.115036 & 0.072276 & -0.114041 \\
\hline & $z$ & 0.062885 & 0.044391 & -0.022735 & 0.257529 \\
\hline \multirow{3}{*}{ Left Chelion } & $x$ & 0.025779 & -0.027681 & 0.030278 & -0.124742 \\
\hline & $y$ & -0.121982 & 0.114939 & 0.085959 & -0.114223 \\
\hline & $z$ & 0.127491 & 0.038363 & 0.030083 & 0.256637 \\
\hline \multirow{3}{*}{ Left Infraorbitale } & $x$ & 0.153162 & 0.204991 & -0.155992 & -0.025605 \\
\hline & $y$ & 0.011816 & -0.066336 & 0.069849 & -0.028775 \\
\hline & $z$ & 0.05999 & 0.024546 & 0.045091 & 0.03005 \\
\hline \multirow{3}{*}{ Left Frontotemporale } & $x$ & 0.116605 & 0.011214 & 0.243554 & -0.059974 \\
\hline & $y$ & 0.184285 & -0.067633 & -0.286911 & 0.050317 \\
\hline & $z$ & 0.021191 & 0.081583 & 0.026568 & -0.150199 \\
\hline \multirow{3}{*}{ Left Zygofrontale } & $x$ & 0.119144 & 0.015247 & 0.290324 & -0.048237 \\
\hline & $y$ & 0.104492 & -0.039884 & -0.209893 & 0.097919 \\
\hline & $z$ & 0.066023 & 0.07948 & -0.091059 & -0.117063 \\
\hline
\end{tabular}


Table 1: Continued.

\begin{tabular}{|c|c|c|c|c|c|}
\hline Face Dimensions & & PC1 & PC2 & PC3 & PC4 \\
\hline \multirow{3}{*}{ Left Bizigomatic } & $x$ & 0.267026 & 0.194492 & -0.025595 & -0.159568 \\
\hline & $y$ & 0.041141 & -0.027079 & 0.028875 & 0.100681 \\
\hline & $z$ & -0.100136 & -0.100385 & 0.361976 & 0.051431 \\
\hline \multirow{3}{*}{ Left Gonion } & $x$ & 0.284425 & 0.16777 & 0.239609 & 0.221905 \\
\hline & $y$ & -0.155504 & 0.305318 & 0.073897 & 0.35161 \\
\hline & $z$ & -0.012303 & 0.053004 & -0.081593 & -0.188544 \\
\hline \multirow{3}{*}{ Left Tragion } & $x$ & 0.234216 & 0.087168 & 0.122075 & -0.178044 \\
\hline & $y$ & 0.051841 & -0.041882 & 0.015991 & -0.093947 \\
\hline & $z$ & -0.099114 & 0.04377 & 0.018098 & 0.007539 \\
\hline \multirow{3}{*}{ Right Interpupilary } & $x$ & -0.105114 & -0.025959 & -0.076164 & -0.00666 \\
\hline & $y$ & 0.035167 & -0.102977 & 0.002433 & -0.063109 \\
\hline & $z$ & 0.015465 & -0.006616 & -0.017302 & 0.009583 \\
\hline \multirow{3}{*}{ Left Interpupilary } & $x$ & 0.064232 & 0.051679 & 0.046711 & -0.042373 \\
\hline & $y$ & 0.036144 & -0.100849 & 0.006195 & -0.081916 \\
\hline & $z$ & 0.085829 & 0.009695 & 0.04579 & 0.009617 \\
\hline \multirow{3}{*}{ Right Nasal Root } & $x$ & -0.04234 & -0.026263 & -0.028729 & 0.047738 \\
\hline & $y$ & 0.044661 & -0.149109 & -0.007595 & -0.078473 \\
\hline & $z$ & 0.0254 & -0.038598 & 0.029479 & -0.071444 \\
\hline \multirow{3}{*}{ Left Nasal Root } & $x$ & 0.00875 & 0.056566 & -0.014386 & 0.024296 \\
\hline & $y$ & 0.042245 & -0.149522 & -0.005162 & -0.072066 \\
\hline & $z$ & 0.052601 & 0.006138 & 0.0221 & -0.097465 \\
\hline \multirow{3}{*}{ Right Alare } & $x$ & -0.104936 & 0.024283 & -0.057818 & -0.11649 \\
\hline & $y$ & -0.018704 & -0.011104 & 0.069058 & -0.012788 \\
\hline & $z$ & 0.038854 & -0.053892 & -0.011676 & 0.017082 \\
\hline \multirow{3}{*}{ Left Alare } & $x$ & 0.04214 & 0.012693 & 0.015318 & 0.033131 \\
\hline & $y$ & -0.024419 & -0.021711 & 0.089439 & -0.007221 \\
\hline & $z$ & 0.107528 & -0.043892 & 0.034216 & 0.090584 \\
\hline \multirow{3}{*}{ Chin } & $x$ & -0.046142 & -0.080757 & 0.022201 & -0.028164 \\
\hline & $y$ & -0.191757 & 0.381253 & -0.03619 & -0.205793 \\
\hline & $z$ & 0.104467 & 0.162404 & -0.067402 & 0.089818 \\
\hline
\end{tabular}

The first principal component score is calculated as follows: $\mathrm{PC} 1=-0.092402 *(X$ coordinate for Right Tragion $)+$ $0.055546 *$ ( $Y$ coordinate for Right Tragion $)-0.226846$ * $(Z$ coordinate for Right Tragion $) \cdots-0.046142 *(X$ coordinate for Chin $)-0.191757 *(Y$ coordinate for Chin $)$ $+0.104467 *(Z$ coordinate for Chin).

For the above equation, only right tragion and chin are shown. The other 24 landmarks are not shown. The eigenvectors for $x, y, z$ coordinates of the 26 landmarks are shown in Table 1 for PC1-4. The superimposed coordinates are not provided due to limited space.

The projections of the form data for the 947 individual configurations onto PCs 1 through 4 are shown in Figure 3. Each point represents a linear combination of 78 coordinates for a single subject. By design, most of the scatter in the data is along the first PC and somewhat less along the second. Variation on higher PCs is reduced, but nonetheless substantial suggesting future research should examine this more closely, and also examine its relationship to respirator fit and function.
The above is a standard analysis and plotting approach for multivariate data, but since this analysis has been driven by the principals of GM that maintain the relationships in physical space amongst the variables, these results can be used to construct hypothetical configurations of landmarks representing arbitrary points in the space of principal components. Since PCA is based on mean-centered data, and the PCs, themselves, are linear combinations of the original coordinate variables, one can construct the configuration at a specific point in PC space by simply multiplying the coefficients for the linear combination of coordinates represented by each PC of interest by the coordinate of the point of interest on that PC. The most common use of this technique is to generate visualizations of the patterns of variation captured by particular PCs. Landmark configurations representing patterns of variation along PC1 magnified by a factor of 100 are shown in Figure 4 . The coefficients are scaled so the sum of their squares equals 1.0. Hence with 78 coefficients, these are small numbers that require considerable magnification. 


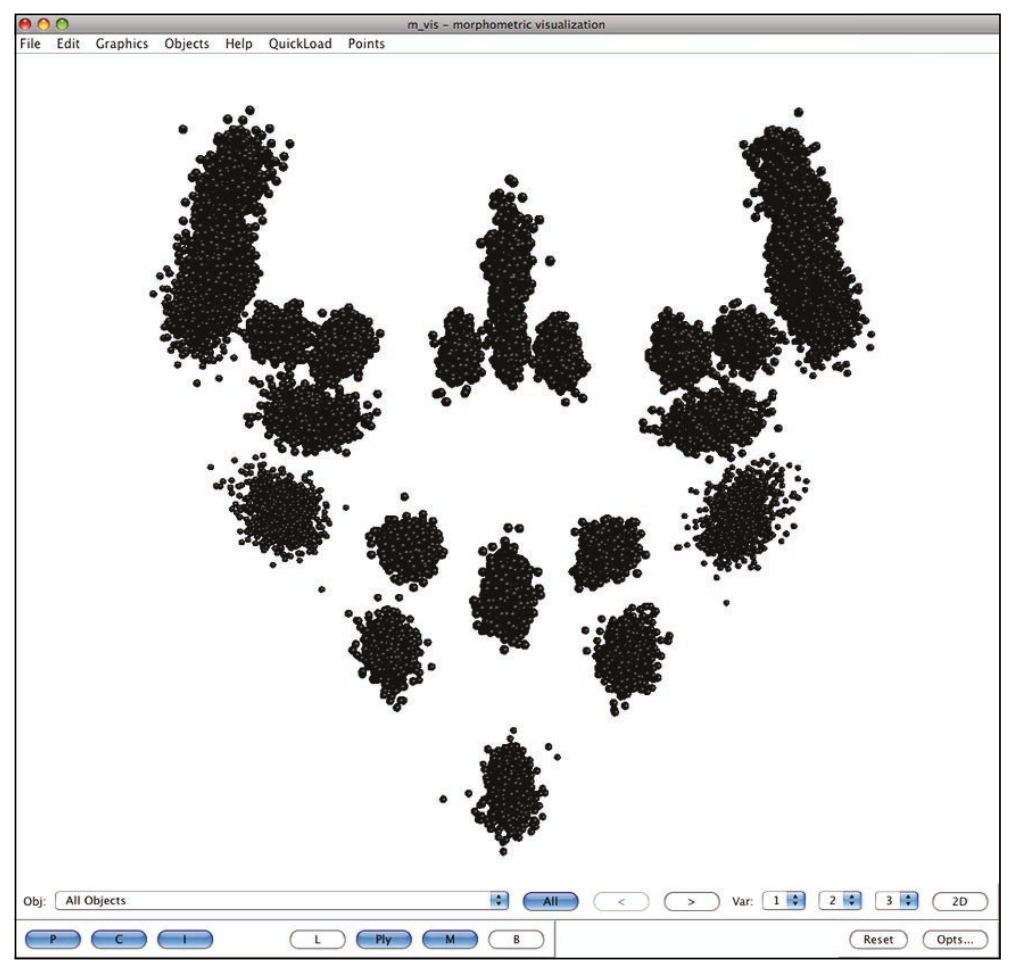

FIGURE 2: Screen shot from the Morpheus et al. software illustrating a frontal scatter plot of the 947 individuals for each of the 26 landmarks in the data set after GPA and size restoration. The coordinates of the 26 landmarks represented for each specimen are form variables that describe the shape and size of each specimen.

The pattern of variation specified by PC1 and shown graphically in Figure 4 shows a general movement of landmarks away from their joint center of gravity in the positive direction along the PC. Shape change in the negative direction is, of course, the compliment of this with landmarks all moving more-or-less toward the configuration's center at approximately the same rate (distance per unit change along the axis). It is important to note that the polarity of these axes is arbitrary and positive and negative directions can be exchanged without impacting the variance of the projections, which is the only criterion by which they are constructed.

Such a pattern clearly represents an overall increase or diminution of the configuration as results from isometric size change. Indeed, the correlation of the scores of individuals on this axis with their centroid size is 0.99 (= Pearson's product moment correlation, Kendall's tau $=0.92)$. Such a result indicates that the overall size is an important component of facial variability in the studied population and is likely an important component of respirator fit assessment, but would not be captured by a standard GM analysis that focuses more on pure shape change. The relatively low proportion of variation (0.26) suggests, however, that size is not the only important consideration.

Figure 5 shows a visualization of facial change in the positive direction of PC2. As before, what represents positive versus negative change along this axis is arbitrary and the negative change in this representation would simply be the reflection of the displacements shown in red along their own axes.

There is a general tendency for landmarks to be displaced medially. The landmarks associated with the upper part of the face, especially those of the eyes and the bridge of the nose, tend to be displaced upwards. Those associated with the lower face-the corners of the mouth, the angle of the jaw, and the chin, tend to be displaced downwards. This has a relatively simple interpretation as those individuals with more positive scores on this axis having relatively narrower and longer faces. Conversely, individuals with more negative scores would have shorter, wider faces. Given the high correlation of the first PC with size, it is not surprising that there is a low association between size and this axis (Pearson's product-moment correlation $=0.09$, Kendall's tau $=0.05)$. This represents independence between overall facial shape (long/narrow versus short/wide) and facial size. In traditional biological terms, this is an indication of a lack of "allometry." Furthermore, this result means that simple concepts of small, medium, and large with respect to respirators cannot capture much of this component of variation.

Together PC1 and 2 represent 36\% of total variation in the data. Thus, even higher PCs may represent patterns of variation that are important components in the general workforce population.

Figure 6 shows the pattern of variation specified by PC3 accounting for about $8 \%$ of the total variation. The 


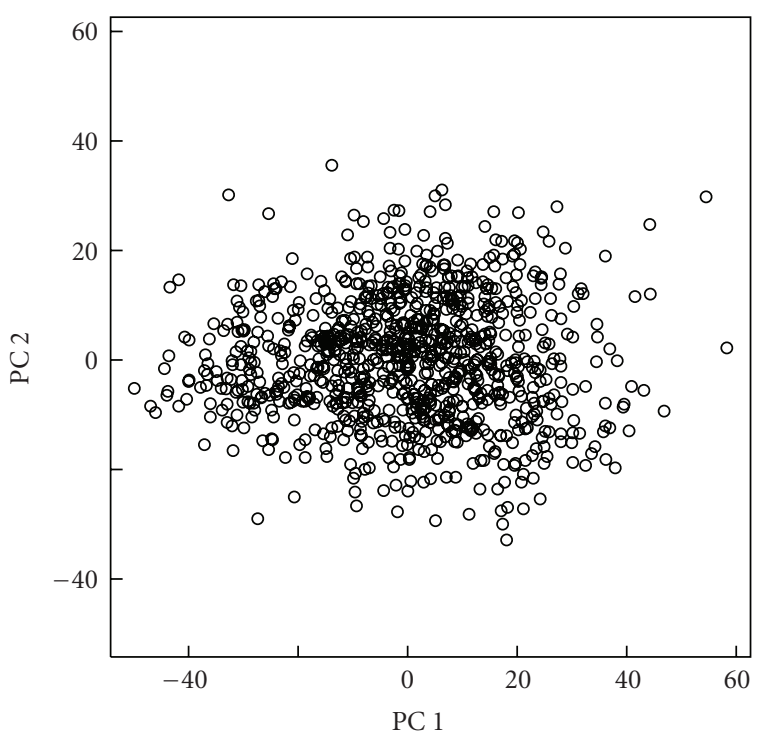

(a)

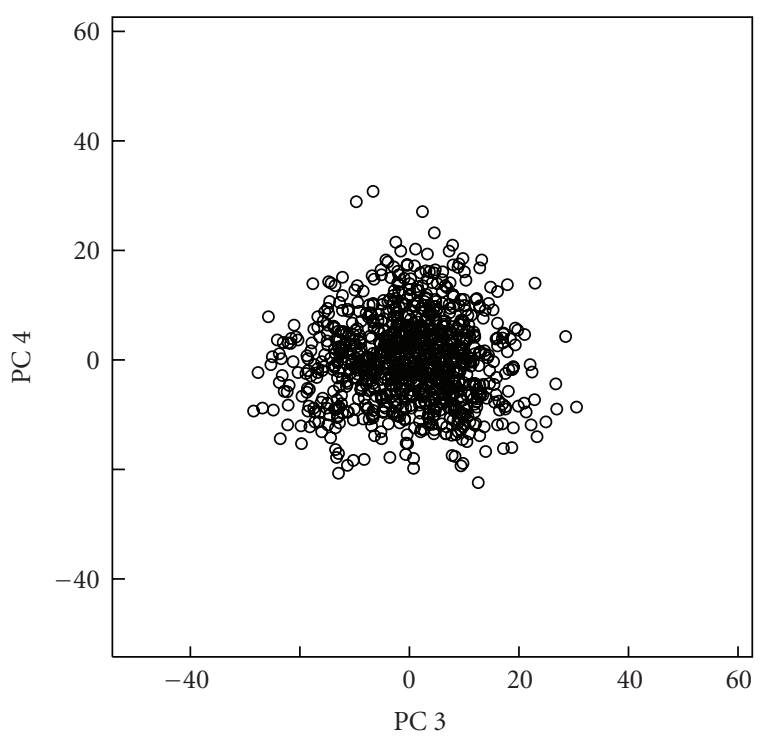

(b)

Figure 3: Projections of 947 landmark configurations onto PCs 1 and 2 (a) and PCs 3 and 4 (b).

pattern here is more complicated and less easy to summarize than those in lower PCs. Important features in the positive direction appear to be a relative lateral displacement of the centers of the pupils and a larger lateral displacement of the landmarks associated with the frontal bone, sides of the head, and angles of the jaw (gonion). In contrast, the landmarks defining the tip and sides of the nose and corners of the mouth are displaced upwards. In seeming contrast, right and left infraorbitale appear medially displaced. In lateral view, gonion and frontotemporale and zygofrontale are displaced posteriorly while zygion shifts anteriorly. This pattern defies simple description, though the nose and mouth do appear to shift superiorly relative to the rest of the face, while the face, itself, appears to widen. Projections on more negative values of this axis, of course, are represented by the compliment of these changes.

The pattern specified by PC4 (Figure 7), though accounting for only $5 \%$ of the total variation, is somewhat more clear. The pupils, nasal root points, the corners of the mouth, and the chin landmarks are shifted inferiorly while gonion is shifted superiorly. Tragion, zygion, frontotemporale, and zygofrontale are shifted medially, and the alare are displaced laterally in frontal view. In lateral view, gonion and the landmarks of the nasal bridge and orbital rim are shifted posteriorly, while the mouth, chin, tragion, and zygion are shifted anteriorly. Configurations projected to more negative scores along this axis manifest the compliment of these changes. In general, there is an impression that this component might represent variation in the degree of ortho/prognathism with positively scoring individuals having longer, wider, and more projecting lower jaws than negatively scoring individuals.

\section{Discussion}

The comprehensive assessment of morphological variation in users may contribute to understanding how differences in facial form can affect the fit and efficacy of commercial respirators. Such knowledge should facilitate the optimal design of these products and inform the development of standards and protocols by which such devices are evaluated and certified. Recent advances in the quantitative analysis of anatomical variation, called geometric morphometric methods, have the potential to provide more powerful and complete descriptions of morphological diversity in a target population than the traditional anthropometric measurements upon which current respirator standards are based. Furthermore, it is important that emerging standards be reflective of an ever-changing workforce that is not likely represented by the military-based standards currently used [5].

The data were carefully checked visually and statistically for incorrect data coding, erroneous values, and other problems that could compromise their use in characterizing relevant morphological variation. Where possible, data coding problems were repaired and erroneous values were marked as missing, and a conservative mean-substitution approach used to impute the coordinate locations. The result was a final, clean data set of 947 individuals for which coordinates for 26 anatomical landmarks were available (either recorded or imputed) for all subjects.

Principal components analysis of variation in the form (size + shape) variables of the data revealed that approximately $26 \%$ of total sample variance could be expressed as a single linear combination of the original variables-PC1. Since this analysis was based on GM methods, the coefficients for this combination could be used to visualize the nature of the captured variation in the physical space of the face. Inspection of the results revealed that the first PC reflected largely isometric size variation. That is, variation in the overall size of faces in the population was the single greatest source of variability within the studied group. 


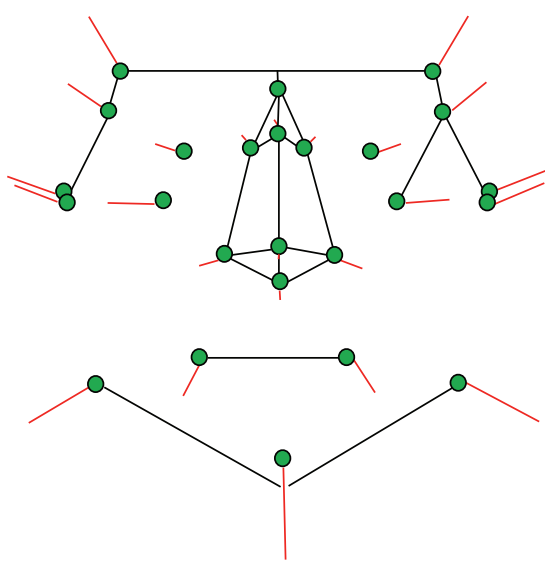

(a)

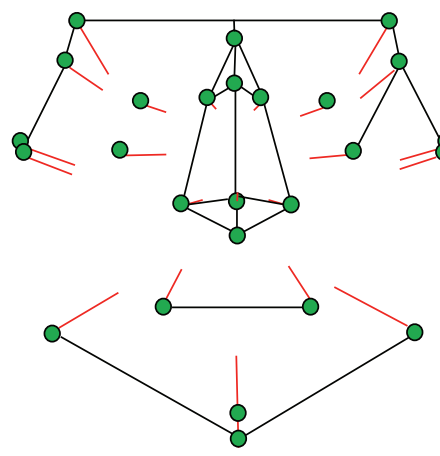

(c)
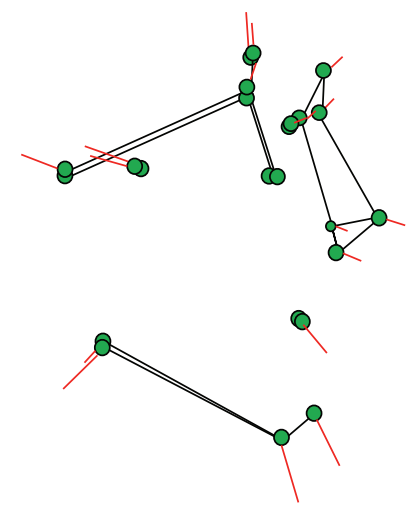

(b)

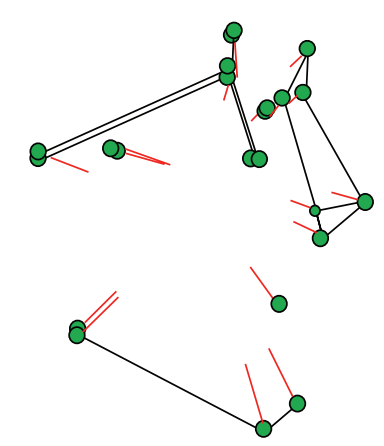

(d)

FIgURE 4: Visualization of PC1. (a) and (c) show frontal view of transformation determined by the first PC. The top is in the positive direction. The bottom is the negative. (b) and (d) are the same, but for the right lateral view. Green circles represent the average location of landmarks in the entire, superimposed data set. The black lines are links to aid visualization. The red line segments represent the coefficients for each coordinate of each landmark specified by the first PC magnified by a factor of 100 to emphasize the pattern of variation. That is, the red lines indicate the path (direction and relative magnitude) of the landmarks as they change location and move along the specified PC in the indicated direction. The ends of the line segments in the images on the top row indicate the positions of the landmarks at a point 100 units out in the positive direction on PC1. The bottom row is the same for 100 units in the negative direction.

While expressing the greatest amount of variation, PC1 does not express most of the variation in the sample and higher PCs may be important in respirator fit research. Visualization of PC2 (expressing about $10 \%$ of sample variation) revealed a contrast between longer, narrower, shallower heads/faces versus shorter, wider, deeper heads/faces that is statistically independent of overall head size. These results for PC1 and PC2 are consistent with the results reported by Zhuang et al., who performed a PCA using 10 linear dimensions related to respirator fit [5]. More complex, but still interpretable and potentially relevant, variation was identified on PC3 ( $\sim \%$ of sample variation) and PC4 $(\sim 5 \%)$.

After analysis, concerns were raised about splits in some of the heads that are the result of movement during the scan. A review of all the scans revealed 109 scans with a split greater than $4 \mathrm{~mm}$. These scans were removed from the PCA so that it could be reanalyzed to see if these aberrations due to movement impacted the results. The resultant PCA showed no statistical difference when compared to the original. Because of this, the information from all heads was retained.
Further study will investigate the correlation between respirator fit and these PCs. This will be done via regression of shape-coordinate and ancillary anthropometric data onto respirator fit measures for 30 test subjects. The result will be a statistical summary and visualization of the components of facial variation most associated with respirator fit. Also, the residuals from the landmark-traditional comparison would be assessed for significant association with the respirator fit data. A significant result may indicate important information captured by the coordinate analysis and missed by the traditional measurements.

The NIOSH anthropometric survey data, respirator fit test panels, and digital 3D headforms have been incorporated into national and international respiratory protection standards $[4,5,16]$. Products certified under these standards are used to protect against chemical, biological, radiological, and nuclear agents for fire fighters and emergency responders. They are also used to protect hospital workers and air travelers from $\mathrm{H} 1 \mathrm{~N} 1$ exposures. If the PC scores are highly correlated to respirator fit, the proposed method in this paper will be applied to develop respirator fit test panels and 


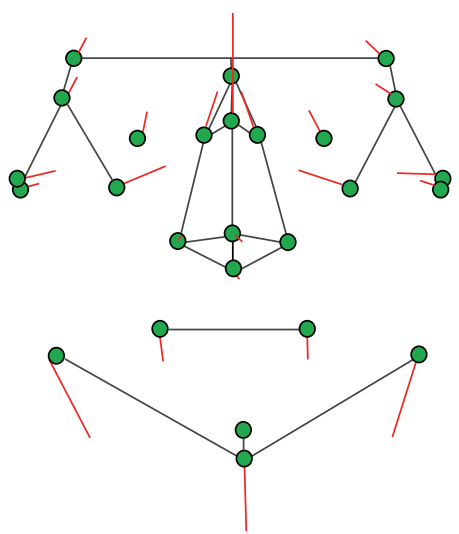

(a)

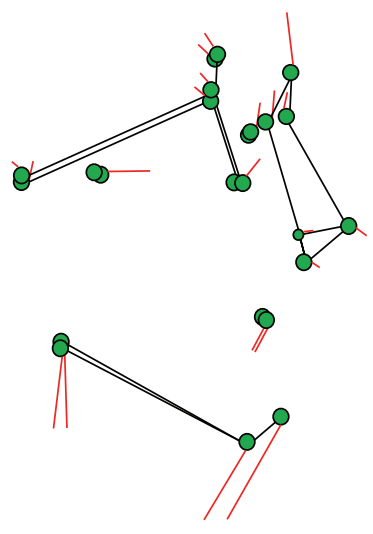

(b)

Figure 5: Visualization of PC2. (a) is frontal view. (b) is lateral view. These are positive-direction displacements. Negative direction is the reflection of all of the red vectors along their own axes.

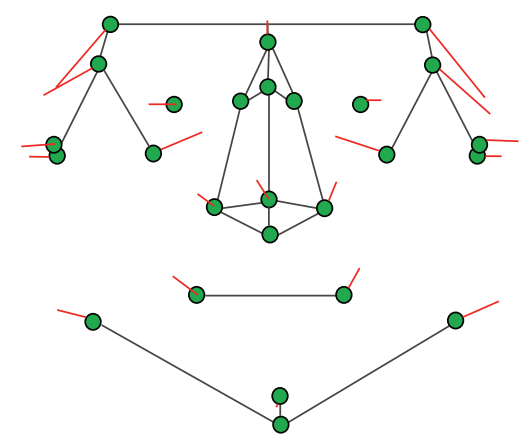

(a)

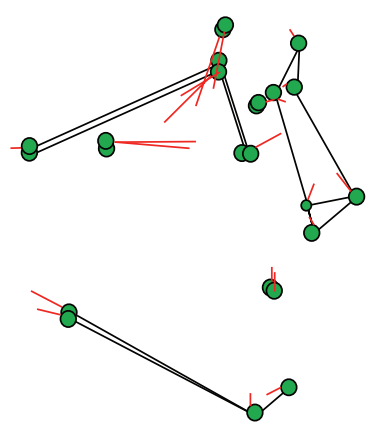

(b)

FIgURE 6: Visualization of PC3. (a) is frontal view. (b) is lateral view. These are positive displacements. Negative direction is the reflection of the red vectors along their own axes.

digital headforms which will in turn be applicable to defense and security.

\section{Conclusions}

In all, these analyses show that the GM-based approach to morphological variation provides a detailed and interpretable assessment of morphological variation in the provided sample that should be very useful in assessing the

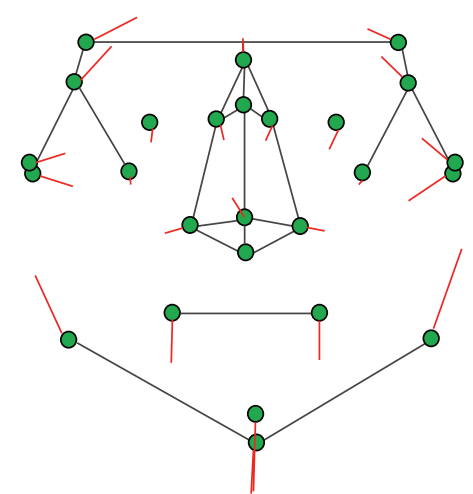

(a)

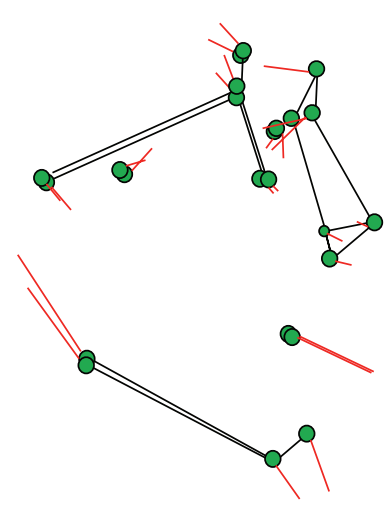

(b)

FIGURE 7: Visualization of PC4. (a) is frontal view. (b) is lateral view. These are positive-direction displacements. Negative direction is the reflection of the red vectors along their own axes.

function of commercial respirators and devising new test and certification standards. A significant amount of this variation is contained in the first few PCs, but a substantial portion remains that could be important in respirator fit. Principal component analysis is not designed to optimize or take into account the results of the respirator fit testing. The relationship between this measure and the results reported here will be the subject of subsequent analyses.

\section{Disclaimer}

The findings and conclusions in this report are those of the authors and do not necessarily represent the views of the National Institute for Occupational Safety and Health. Mention of commercial product or trade name does not constitute endorsement by the National Institute for Occupational Safety and Health.

\section{Acknowledgment}

Ms. S. Lynch performed this research while holding a National Research Council Resident Research Associateship at the National Institute for Occupational Safety and Health (NIOSH), National Personal Protective Technology Laboratory (NPPTL). 


\section{References}

[1] A. L. Hack, E. C. Hyatt, B. J. Held, T. D. Moore, C. P. Richards, and J. T. McConville, Selection of Respirator Test Panels Representative of U.S. Adult Facial Sizes, Los Alamos Scientific Laboratory, Los Alamos, NM, USA, 1974.

[2] A. L. Hack and J. T. McConville, "Respirator protection factors-part I: development of an anthropometric test panel," American Industrial Hygiene Association Journal, vol. 39, no. 12, pp. 970-975, 1978.

[3] Z. Zhuang, J. Guan, H. Hsiao, and B. Bradtmiller, "Evaluating the representativeness of the LANL respirator fit test panels for the current U.S. civilian workers," Journal of the International Society for Respiratory Protection, vol. 21, pp. 83-93, 2004.

[4] Z. Zhuang and B. Bradtmiller, "Head-and-face anthropometric survey of U.S. respirator users," Journal of Occupational and Environmental Hygiene, vol. 2, no. 11, pp. 567-576, 2005.

[5] Z. Zhuang, B. Bradtmiller, and R. E. Shaffer, "New respirator fit test panels representing the current U.S. civilian work force," Journal of Occupational and Environmental Hygiene, vol. 4, no. 9, pp. 647-659, 2007.

[6] D. E. Slice, "Modern morphometrics," in Modern Morphometrics in Physical Anthropology, D. E. Slice, Ed., Kluwer Academic/Plenum Publishers, New York, NY, USA, 2005.

[7] D. E. Slice, "Geometric morphometrics," Annual Review of Anthropology, vol. 36, pp. 261-281, 2007.

[8] J. C. Bailar III, E. A. Meyer, and R. Pool, Eds., Assessment of the NIOSH Head-and-Face Anthropometric Survey of $U$. $S$. Respirator Users, Institute of Medicine of the National Academies. National Academies Press, Washington, DC, USA, 2007.

[9] D. Burnsides, P. M. Files, and J. J. Whitestone, "INTEGRATE 1.25: a prototype for evaluating three-dimensional visualization, analysis, and manipulation functionality," Tech. Rep. AL/CF-TR-1996-0095, Crew Systems Directorate, Human Engineering Division, Wright-Patterson AFB, Dayton, Ohio, USA, 1996.

[10] R. J. A. Little and D. B. Rubin, Statistical Analysis with Missing Data, John Wiley \& Sons, New York, NY, USA, 1987.

[11] J. C. Gower, "Generalized procrustes analysis," Psychometrika, vol. 40, no. 1, pp. 33-51, 1975.

[12] F. J. Rohlf and D. E. Slice, "Extensions of the Procrustes method for the optimal superimposition of landmarks," Systematic Zoology, vol. 39, pp. 40-59, 1990.

[13] F. L. Bookstein, Morphometric Tools for Landmark Data: Geometry and Biology, Cambridge University Press, New York, NY, USA, 1991.

[14] R Development Core Team, "R: a language and environment for statistical computing," R Foundation for Statistical Computing, Vienna, Austria, 2007, http://www.R-project.org/.

[15] D. E. Slice, Morpheus et al.: Software for Morphometric Research. Revision 01-31-00, Department of Ecology and Evolution, State University of New York, Stony Brook, NY, USA, 1998.

[16] Z. Zhuang, S. Benson, and D. J. Viscusi, "Digital 3-D headforms with facial features representative of the current U.S. work force," Ergonomics, vol. 53, no. 5, 2010. 